\title{
Multicenter phase II study of trastuzumab plus S-1 alone in elderly patients with HER2-positive advanced gastric cancer (JACCRO GC-06)
}

\author{
Yutaka Kimura $^{1} \cdot$ Masashi Fujii $^{2} \cdot$ Toshiki Masuishi $^{3} \cdot$ Kazuhiro Nishikawa $^{4} \cdot$ Chikara Kunisaki $^{5} \cdot$ Satoshi Matsusaka $^{6}$. \\ Yoshihiko Segawa ${ }^{7} \cdot$ Masato Nakamura $^{8} \cdot$ Kinro Sasaki $^{9} \cdot$ Narutoshi Nagao $^{10} \cdot$ Yukimasa Hatachi $^{11} \cdot$ Yasuhiro Yuasa $^{12}$. \\ Shinya Asami ${ }^{13}$. Masahiro Takeuchi ${ }^{14}$. Hiroshi Furukawa ${ }^{1}$. Toshifusa Nakajima ${ }^{2}$ on behalf of the JACCRO GC-06 \\ Study Group
}

Received: 30 June 2017 / Accepted: 8 September 2017 / Published online: 21 September 2017

(c) The Author(s) 2017. This article is an open access publication

\begin{abstract}
Background S-1 plus cisplatin is a standard regimen for advanced gastric cancer (AGC) in Asia. The ToGA trial established a fluoropyrimidine plus cisplatin and trastuzumab as a standard treatment for human epidermal growth factor receptor 2 (HER2)-positive AGC. In the HERBIS-1 trial, trastuzumab combined with S-1 plus cisplatin showed promising antitumor activity in patients with HER2-positive AGC. However, cisplatin has several important drawbacks, including vomiting and renal toxicity. These disadvantages of cisplatin are prominent in elderly patients. Therefore, we conducted a prospective phase II study of trastuzumab plus S-1 without cisplatin in elderly patients with HER2-positive AGC.

Methods Patients 65 years or older who had HER2-positive AGC received S-1 orally on days 1-28 of a 42-day cycle and trastuzumab intravenously on day 1 of a 21-day cycle.

Results A total of 51 patients were enrolled. Two patients were ineligible. The full analysis set thus comprised 49 patients. The median age was 71 years (range 65-85). The confirmed response rate was $40.8 \%$ (95\% CI 27.1-54.6\%), and the null hypothesis was rejected. The median follow-up period was 10.6 months. Median overall survival was 15.8 months. Median progression-free survival was 5.1 months, and time to treatment failure was 4.0 months. Major grade 3 or 4 adverse events included neutropenia (12.0\%), anemia (24.0\%), diarrhea (10.0\%), and anorexia (12.0\%). There was one treatment-related death.

Conclusions Trastuzumab in combination with S-1 alone demonstrated promising antitumor activity and manageable toxic effects as well as promising survival results in elderly patients with HER2-positive AGC.
\end{abstract}

Clinical trials registration UMIN000007368.

Keywords Gastric cancer $\cdot$ Elderly patients $\cdot$ HER2-positive $\cdot$ S- $1 \cdot$ Trastuzumab

The original version of this article was revised: The twelfth author name was changed as "Yasuhiro Yuasa".

The JACCRO GC-06 Study Group Investigators are listed in the "Acknowledgements" section.

Electronic supplementary material The online version of this article (doi:10.1007/s10120-017-0766-x) contains supplementary material, which is available to authorized users.

Masashi Fujii

gioncology2006@yahoo.co.jp

Extended author information available on the last page of the article

\section{Background}

Gastric cancer is the second most common cause of cancerrelated death worldwide [1]. The only potentially curative treatment is surgical resection. However, regional and distant recurrence often occurs after surgery. The standard treatment for advanced or recurrent gastric cancer (AGC) is chemotherapy, given in hope of prolonging survival.

A global standard regimen for AGC is a fluoropyrimidine plus a platinum compound. In Western countries, docetaxel or epirubicin is usually added to this regimen $[2,3]$. In East Asia, an oral fluoropyrimidine, S-1, has become the most widely used drug for the treatment for AGC [4], and S-1 plus cisplatin is regarded as a standard 
regimen on the basis of the results of the phase III SPIRITS trial comparing S-1 plus cisplatin with S-1 alone for the first-line treatment of AGC [5].

Recently, the ToGA (Trastuzumab in Combination with Chemotherapy vs. Chemotherapy Alone for Treatment of HER2-Positive Advanced Gastric or Gastro-oesophageal Junction Cancer) study, an international phase III trial, evaluated trastuzumab, a humanized monoclonal antibody that blocks human epidermal growth factor receptor-2 (HER2) activation, in combination with capecitabine and cisplatin. This study demonstrated that the addition of trastuzumab provided a significant survival benefit, which was much greater in patients whose tumors highly overexpressed HER2 [immunohistochemistry (IHC), 3+ or 2+ ; fluorescent in situ hybridization (FISH) positive] [6].

A phase II trial of trastuzumab combined with S-1 plus cisplatin, a standard regimen in East Asia, was performed in patients who had AGC with high HER2 overexpression (HERBIS-1 study) [7]. The HERBIS-1 study demonstrated a high response rate (RR 68\%) and prolonged survival (OS 16.0 months). Subsequently, S-1 plus cisplatin and trastuzumab became a standard regimen, as did capecitabine plus cisplatin and trastuzumab, for highly HER2-positive AGC in Japan.

However, cisplatin has several important drawbacks, including high incidences of nausea, vomiting [8], and renal toxicity $[9,10]$, the need for admission to receive treatment, and other adverse events negatively affecting the quality of life (QoL) of patients. Cisplatin is contraindicated in patients with poor renal function. These disadvantages of cisplatin are particularly problematic in elderly patients. Importantly, gastric cancer is a disease of aging, so the risk of it increases with advancing age.

We thus conducted this phase II study to evaluate the efficacy and safety of S-1 alone plus trastuzumab without cisplatin in elderly patients with highly HER2-positive AGC.

\section{Methods}

\section{Study design}

The Japan Clinical Cancer Research Organization (JACCRO) GC-06 study was a multicenter, prospective, phase II trial. The study was performed in accordance with the Declaration of Helsinki and Ethical Guidelines for Clinical Studies in Japan. The protocol was approved by the ethics committees of JACCRO and of each participating center before initiating enrollment. An Independent Data Monitoring Committee reviewed all efficacy and safety data.

\section{Patients}

Eligible patients had to have a histologically confirmed diagnosis of gastric or esophagogastric junction adenocarcinoma, measurable disease according to the Response Evaluation Criteria in Solid Tumors (RECIST) version 1.1, and highly HER2-positive cancer as confirmed by immunohistochemistry (IHC), fluorescence in situ hybridization (FISH) (IHC $3+$ or IHC2+ and FISH positive), or both. Eligible patients also had to fulfill all of the following conditions: an age of 65 years or older at the time of obtaining informed consent; an Eastern Cooperative Oncology Group (ECOG) performance status of 0,1 , or 2 ; adequate organ function (a leukocyte count between 3500 and $12,000 / \mathrm{ml}$, a neutrophil count at least $2,000 / \mathrm{ml}$, hemoglobin level at least $9.0 \mathrm{~g} / \mathrm{dl}$, a platelet count at least $100,000 / \mathrm{ml}$, a serum bilirubin level of no more than $1.5 \mathrm{mg} / \mathrm{dl}$, serum aspartate aminotransferase and alanine aminotransferase levels of no more than $100 \mathrm{IU}$; serum creatinine level less than $1.2 \mathrm{mg} / \mathrm{dl}$, creatinine clearance no more than $50 \mathrm{ml} / \mathrm{min}$ ); a left ventricular ejection fraction (LVEF) of $\geq 50 \%$ as measured on echocardiography or multiple gated acquisition (MUGA) scanning within 21 days before enrollment; no prior chemotherapy or radiotherapy for gastric cancer; and an expected survival of at least 3 months. Written informed consent was obtained from all patients.

\section{Treatment}

Patients received S-1 (80-120 mg per day) orally on days 1-28 of a 42-day cycle and trastuzumab (first dose $8 \mathrm{mg}$ / $\mathrm{kg}$; second dose onward $6 \mathrm{mg} / \mathrm{kg}$ ) intravenously on day 1 of a 21-day cycle (the criteria for withholding, resuming, and dose reduction of S-1 and trastuzumab, and the modification of treatment with S-1 are shown in the table in the "Electronic supplementary material").

\section{Outcomes}

The primary endpoint of this study was the response rate (RR). The secondary endpoints were overall survival (OS), progression-free survival (PFS), time to treatment failure (TTF), and safety. Tumors were measured every 6 weeks by the investigators at each participating center until the onset of progressive disease. An extramural review committee assessed all images obtained in the study according to the RECIST. The OS was defined as the time from the date of enrollment to the date of death from any cause. PFS was defined as the time from the date of enrollment to the date of disease progression or death from any cause. TTF was defined as the time from the date of enrollment 
to the date when the treating physician decided to discontinue treatment for any reason. Physical examinations and blood tests were mandatory before each course of treatment, and LVEF was assessed every 3 months during treatment. Adverse events were evaluated according to the National Cancer Institute Common Terminology Criteria for Adverse Events, version 4.0.

\section{Statistical analysis}

The reported RR of S-1 alone in elderly patients with AGC was 14.3-21.7\% [11-13] and the RR of fluoropyrimidine plus cisplatin was reported $35 \%$ by ToGA trial. Then, the required sample size was estimated on the basis of a threshold RR of $20 \%$ and an expected RR of 35\%, 80-90\% power, and an alpha value of 0.1 (one-sided) using the binomial test. Due to eventual ineligible patients, the target sample size was determined to be at least 40 patients at a power of $80 \%$. Efficacy was evaluated in all patients who received the study treatment. We used the Kaplan-Meier method to estimate survival curves and Greenwood's formula to calculate $95 \%$ confidence intervals (CI) for survival rates. Statistical analyses were conducted with SAS software, version 9.2.

\section{Results}

\section{Patient disposition and characteristics}

Patients were enrolled from March 2012 through February 2014. The last follow-up analysis was conducted in March 2016. Forty patients were enrolled before completion of the planned accrual period of 2 years, increasing the power of this study from 80 to $90 \%$. Finally, 51 patients were enrolled in the study within 2 years at 24 centers in Japan. However, two patients were ineligible because they lacked measurable lesions or could not receive the protocol treatment. A total of 50 patients, including the patient with no measurable lesion, were included in safety analyses, and the full analysis set comprised 49 patients (actual power $87 \%$ ). The characteristics of the 49 patients are shown in Table 1 . The median age was 71 years (range 65-85), and the ECOG performance status was 0 in 32 patients, 1 in 14 patients, and 2 in 3 patients. Thirty-seven patients (76\%) had differentiated adenocarcinoma. Forty-one patients (84\%) had unresectable lesions, and 8 patients (16\%) had recurrent disease. The most frequent site of metastasis was lymph nodes (78\%), followed by the liver (45\%), lung (14\%), and peritoneum (14\%). The proportions of IHC $3+$ and IHC $2+/$ FISH-positive tumors were 71 and $29 \%$, respectively.
Table 1 Patient characteristics

\begin{tabular}{|c|c|}
\hline Characteristics & $n=49$ \\
\hline \multicolumn{2}{|l|}{ Age (years) } \\
\hline Median & 71 \\
\hline Range & $65-85$ \\
\hline \multicolumn{2}{|l|}{ Gender } \\
\hline Male & $36(73.5 \%)$ \\
\hline Female & $13(26.5 \%)$ \\
\hline \multicolumn{2}{|l|}{ ECOG PS } \\
\hline 0 & $32(65.3 \%)$ \\
\hline 1 & $14(28.6 \%)$ \\
\hline 2 & $3(6.1 \%)$ \\
\hline \multicolumn{2}{|l|}{ Histological type } \\
\hline Differentiated & $37(75.5 \%)$ \\
\hline Undifferentiated & $12(24.5 \%)$ \\
\hline \multicolumn{2}{|l|}{ Previous gastrectomy } \\
\hline No & $36(73.5 \%)$ \\
\hline Yes & $13(26.5 \%)$ \\
\hline \multicolumn{2}{|l|}{ Unresectable/recurrent } \\
\hline Unresectable & $41(83.7 \%)$ \\
\hline Recurrent without adjuvant chemotherapy & $5(10.2 \%)$ \\
\hline Recurrent with adjuvant chemotherapy & $3(6.1 \%)$ \\
\hline \multicolumn{2}{|l|}{ Metastatic sites } \\
\hline Lymph nodes & $38(77.6 \%)$ \\
\hline Liver & $22(44.9 \%)$ \\
\hline Lung & $7(14.3 \%)$ \\
\hline Peritoneum & $7(14.3 \%)$ \\
\hline \multicolumn{2}{|l|}{ HER2 status } \\
\hline IHC3+ & $35(71.4 \%)$ \\
\hline IHC2+ and FISH-positive & $14(28.6 \%)$ \\
\hline
\end{tabular}

ECOG PS Eastern Cooperative Oncology Group performance status, IHC immunohistochemistry

\section{Efficacy}

All patients were treated on an outpatient basis. The median number of treatment cycles of S-1 was 3.0 (range 1-29), and the median relative dose intensity was $90.4 \%$ for S-1 and $100 \%$ for trastuzumab. At the time of analysis, one patient was still receiving treatment, and all other patients had discontinued treatment. The main reason for discontinuation of treatment was progressive disease (37 patients). Only 5 patients with thrombocytopenia, pneumothorax, ileus, handfoot syndrome and febrile neutropenia discontinued treatment, and 2 patients with para-aortic lymph node metastasis after $2-3$ cycles of treatment underwent surgery because of a significant response.

The confirmed RR [complete response (CR), 2 patients; partial response (PR), 18 patients; stable disease (SD), 21 patients; progressive disease (PD), 8 patients] was $40.8 \%$ (95\% CI $27.1-54.6 \%$; $80 \%$ CI $31.8-49.8 \%$ ); therefore, the 
null hypothesis for the primary end point $(\mathrm{RR}=20 \%)$ was rejected. The confirmed RR was $40.5 \%$ (95\% CI 24.7-56.4\%) in the patients with differentiated cancer $(n=37)$ and $41.7 \%$ (95\% CI 13.8-69.6\%) in the patients with undifferentiated cancer $(n=12)$. Among the 20 patients with CR or PR, the median time to response and duration of response were 43 days (range 27-120 days) and 134 days (range 43-1152 days), respectively. The disease control rate (i.e., the proportion of patients who had CR, PR, or SD) was $83.7 \%$ (95\% CI 73.3-94.0\%). A waterfall plot of the confirmed best overall response for each patient is shown in Fig. 1. The median duration of follow-up for the 49 patients at the time of analysis (March 2016) was 10.6 months. The median OS was 15.8 months (95\% CI 9.2-20.4), and the 1-year OS rate was $56.9 \%$ (95\% CI 41.4-69.7\%; Fig. 2). The median PFS was 5.1 months (95\% CI 3.8-5.6), and the 1-year PFS rate was $15.9 \%$ (95\% CI 6.5-29.1\%; Fig. 3). The median TTF was 4.0 months (95\% CI 3.1-5.4).

There was no statistically significant difference between patients with IHC $3+(n=35)$ and IHC2+/FISH-positive $(n=14)$ status, but the RR of IHC $3+$ was higher than that of IHC2+ and FISH-positive status ( 48.6 vs. $21.4 \%, p=0.11$ ), and overall survival was longer in IHC $3+$ than in IHC2 and FISH positive status (17.5 vs. 8.0 months, $p=0.36$ ).

\section{Safety}

All adverse events are summarized in Table 2. Among the hematological adverse events, the rates of grade 3 or higher neutropenia and anemia were 12 and $24 \%$, respectively. The most frequent common nonhematological toxicity was anorexia (any grade $66 \%$; grade 3 or higher $12 \%$ ).

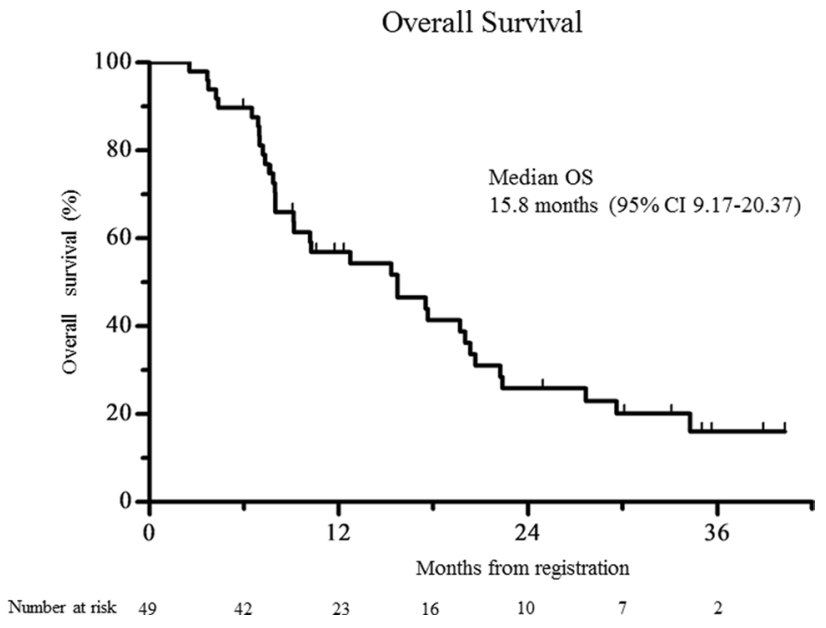

Fig. 2 The Kaplan-Meier overall survival

There was no grade 3 or higher nausea or vomiting. The incidence of elevations of the creatinine level of any grade was only $10 \%$, and there was no grade 3 or higher renal toxicity. Grade 3 infusion-related reactions occurred in 1 patient $(2 \%)$. Heart failure did not occur in any patient. One patient was evaluated to have died from treatmentrelated causes. This patient was an 80 -year-old man with peritoneal metastasis from AGC. There was no severe adverse event during the prior four courses of S-1 plus trastuzumab. Nine days after the fifth dose of trastuzumab, he was found dead at his home. The reason for sudden death was unknown, but we considered the death to be treatment-related because the patient died within 30 days after the last infusion of trastuzumab.

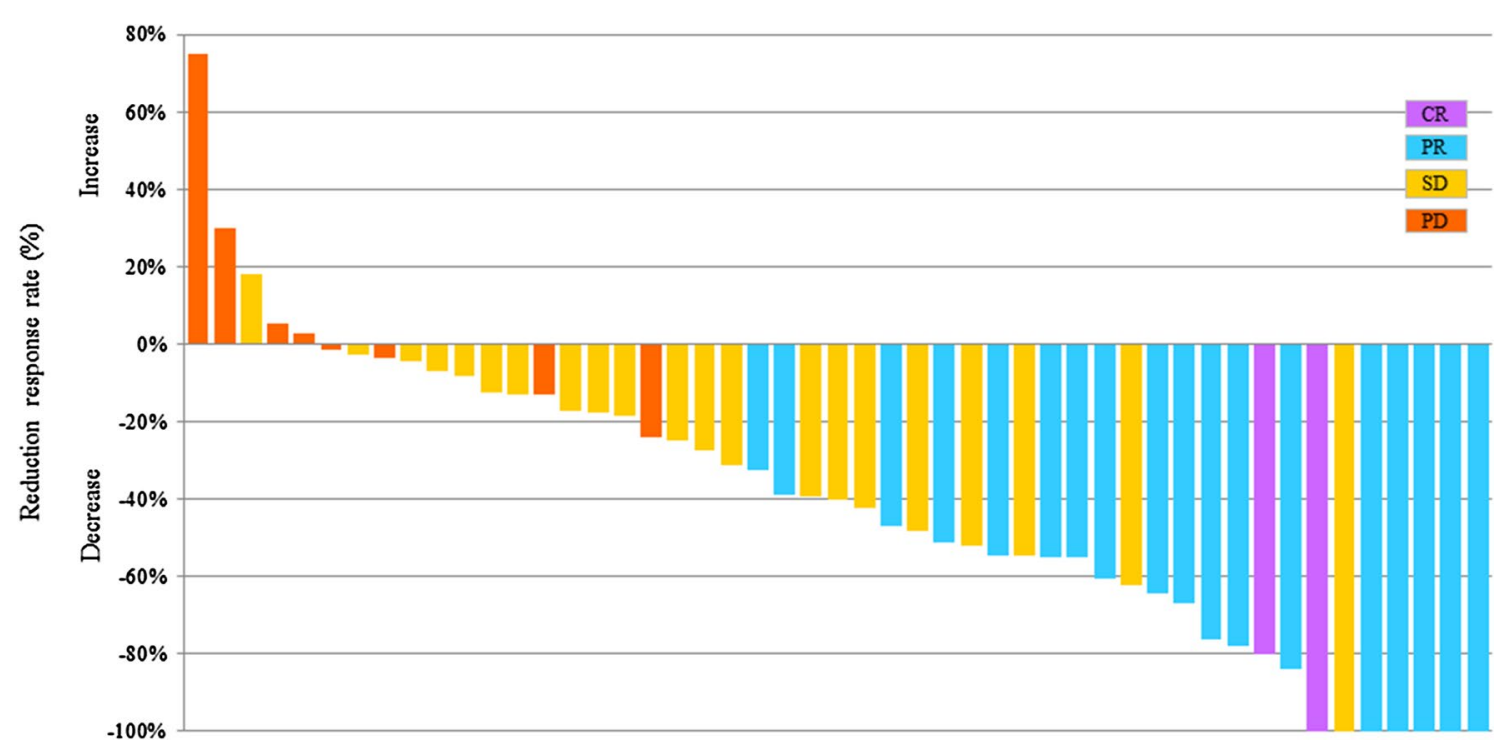

Fig. 1 A waterfall plot showing the confirmed best overall response for each patient 


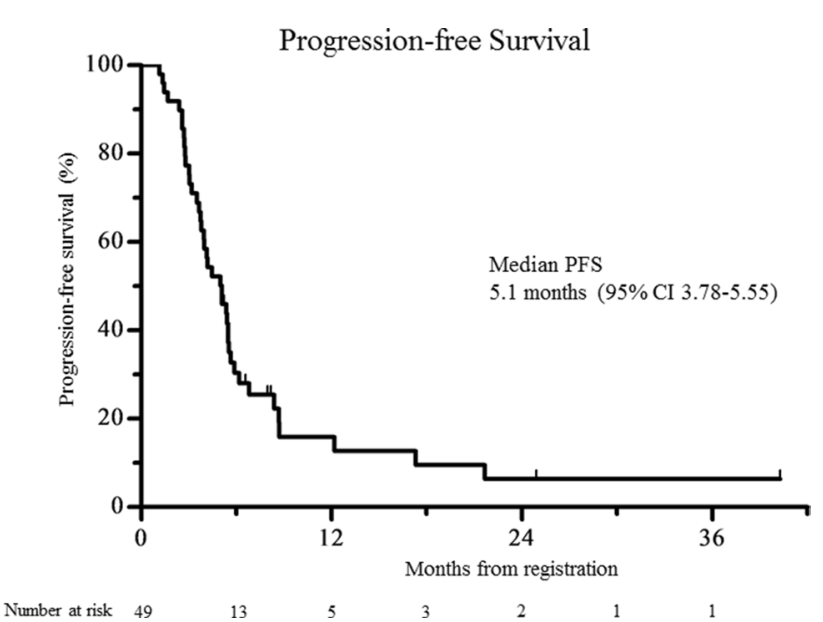

Fig. 3 The Kaplan-Meier progression-free survival

\section{Discussion}

Cancer in the elderly is rapidly increasing worldwide. More than $60 \%$ of patients with gastric cancer are older than 65 years at the time of diagnosis, and about one-third are older than 75 years [14]. Systemic chemotherapy has improved OS and the QoL as compared with supportive care alone in patients with AGC [15-17]. The chronologic age alone is an inadequate reason for avoiding the use of effective cancer treatment that can improve QoL or OS. However, cytotoxic agents such as cisplatin often negatively affect the QoL of elderly patients. A subgroup analysis of the SPIRITS trial reported that the HR of elderly patients was worse than that of younger patients (younger than 60 years of age: HR $0.75 ; 60-69$ years of age: HR $0.98 ; 70-74$ years of age: HR 0.95) [5]. On the other hand, a subgroup analysis of the ToGA study reported that the HR was 0.84 in patients younger than 60 years, as compared with 0.66 in patients 60 years or older, and adding trastuzumab to chemotherapy did not increase adverse events [6]. Therefore, we hypothesized that cisplatin would not prolong survival in elderly patients with tumors overexpressing HER2, and that combining trastuzumab with chemotherapy should be considered. This multicenter phase II study demonstrated that a fluoropyrimidine plus trastuzumab without cisplatin has favorable efficacy and lower toxicity in elderly patients with HER2-positive AGC.

In our study, the RR and disease control rate (DCR) were 40.8 and $83.7 \%$, respectively. The RR in our study was lower than those in the ToGA (47\%) and HERBIS-1 (68\%) studies. However, the DCR was comparable to those in the ToGA (79\%) and HERBIS-1 (94\%) studies. The OS of patients who received a fluoropyrimidine plus trastuzumab without cisplatin was 15.8 months. This survival benefit was similar to those in the HERBIS-1 (16.0 months) and ToGA (highly overexpressing HER2-positive patients,
Table 2 Adverse events

\begin{tabular}{lllllrr}
\hline Event & $n=50$ & & & & Any (\%) & Grade 3-4 (\%) \\
\cline { 2 - 5 } & 1 & 2 & 3 & 4 & & \\
\hline Leukopenia & 10 & 10 & 5 & 0 & 50.0 & 10.0 \\
Neutropenia & 6 & 5 & 6 & 0 & 34.0 & 12.0 \\
Febrile neutropenia & - & - & 1 & 0 & 2.0 & 2.0 \\
Anemia & 6 & 12 & 12 & 0 & 60.0 & 24.0 \\
Thrombocytopenia & 18 & 1 & 1 & 0 & 40.0 & 2.0 \\
AST increased & 16 & 3 & 0 & 0 & 38.0 & 0.0 \\
ALT increased & 10 & 0 & 0 & 0 & 20.0 & 0.0 \\
Creatinine increased & 5 & 0 & 0 & 0 & 10.0 & 0.0 \\
Bilirubin increased & 7 & 3 & 2 & 0 & 24.0 & 4.0 \\
Hypoalbuminemia & 5 & 14 & 3 & 0 & 44.0 & 6.0 \\
Diarrhea & 16 & 4 & 5 & 0 & 50.0 & 10.0 \\
Oral mucositis & 10 & 4 & 4 & 0 & 36.0 & 8.0 \\
Anorexia & 5 & 22 & 6 & 0 & 66.0 & 12.0 \\
Fatigue & 10 & 4 & 1 & - & 30.0 & 2.0 \\
Nausea & 14 & 8 & - & - & 44.0 & 0.0 \\
Vomiting & 11 & 4 & 0 & 0 & 30.0 & 0.0 \\
Infusion-related reaction & 0 & 0 & 1 & 0 & 2.0 & 2.0 \\
Hypertension & 8 & 9 & 2 & 0 & 38.0 & 4.0 \\
Palmar-plantar erythrodyses- & 4 & 2 & 2 & - & 16.0 & 4.0 \\
thesia syndrome & & & & & & \\
\hline
\end{tabular}


16.0 months) studies. On the other hand, the PFS of patients who received a fluoropyrimidine plus trastuzumab without cisplatin was 5.1 months, which was much shorter than those in the HERBIS-1 (7.8 months) and ToGA (6.7 months) studies. We attribute the long survival time after the onset of disease progression to the fact that $65.3 \%$ of patients received second-line chemotherapy, among whom $59.4 \%$ consecutively received trastuzumab. The toxicities of a fluoropyrimidine plus trastuzumab without cisplatin were very mild, as we had expected. In our study, the incidence of grade 3 or higher neutropenia was 12\% (HERBIS-1 36\%; ToGA $27 \%$ ), febrile neutropenia was $2 \%$ (HERBIS-1 $4 \%$; ToGA $5 \%$ ), grade 3 or higher vomiting was $0 \%$ (HERBIS- $16 \%$; ToGA $6 \%$ ), and a grade 3 or higher increased creatinine level was $0 \%$ (HERBIS-1 6\%; ToGA 1\%). These mild toxicities of a fluoropyrimidine plus trastuzumab without cisplatin as the first-line treatment for HER2-positive AGC may have had a positive effect on the introduction of second-line treatment. At the start of the second-line treatment, ECOG performance status was 0 in 17 patients, 1 in 15 patients, 2 in 2 patients, and 3 in 1 patient. The incidence of grade 3 or higher anemia in our study was 24\% (HERBIS-1 $15 \%$; ToGA $12 \%)$, which was significantly higher than that in the ToGA study $(p=0.003)$. Anemia is easy controlled, but should be effectively managed in elderly patients who receive S-1. All patients could receive treatment on an outpatient basis without being admitted to the hospital. We believe that this factor also contributed substantially to the QoL of the elderly patients.

This study had some limitations. Although the PFS was shorter and the OS was almost the same in HERBIS-1 compared to the ToGA study, no details regarding post-progression treatment were provided for HERBIS-1.

\section{Conclusion}

We conclude that trastuzumab plus S-1 alone without cisplatin is an effective and safe treatment that can prolong survival in elderly patients with HER2-positive AGC.

\footnotetext{
Acknowledgements We thank all of the patients and their families, as well as all of the site investigators. A list of the participating institutions is provided below, and is available at Gastric Cancer online. We are deeply grateful to the late Professor Hiroya Takiuchi, who played a pivotal role as a principal investigator at the start of this study. We are also particularly grateful to Yasuhiro Shimada of the Kochi Health Sciences Center, Tsuneo Sasaki of the Tokyo Metropolitan Cancer and Infectious Disease Center Komagome Hospital, and Toru Takebayashi of Keio University for their kind advice as members of the Independent Data Monitoring Committee. We also thank Atsushi Kohno of the Cancer Institute Ariake Hospital and Hiroshi Takahashi of Showa University Fujigaoka Hospital for serving as members of the Extramural Review Committee.

Investigators: Tomono Kawase, Ryohei Kawabata, Yutaka Kimura (Sakai City Medical Center, Sakai); Tetsuya Etou, Toshiki Masuishi
}

(Tsuchiura Kyodo General Hospital, Tsuchiura); Hirochika Makino, Hidetaka Ono, Yusuke Izumisawa, Chikara Kunisaki (Yokohama City University Medical Center, Yokohama); Kazuhiro Nishikawa, Junji Kawada (Osaka General Medical Center, Osaka); Satoshi Matsusaka (Cancer Institute Hospital of the Japanese Foundation for Cancer Research, Tokyo); Ken Shimada, Yu Sunakawa, Yoshihiko Segawa (Saitama Medical University International Medical Center, Hidaka); Masato Nakamura (Aizawa Hospital, Matsumoto); Hitoshi Satomura, Kinro Sasaki (Dokkyo Medical University Hospital, Shimotsugagun); Narutoshi Nagao, Katsuyuki Kunieda (Gifu Prefectural General Medical Center, Gifu); Akihito Tsuji, Hironaga Satake, Yukimasa Hatachi (Kobe City Medical Center General Hospital, Kobe); Hisashi Ishikura, Yasuhiro Yuasa, Hiroshi Okitsu (Tokushima Red Cross Hospital, Komatsushima); Shinya Asami (Fukuyama City Hospital, Fukuyama); Takahiro Ito, Kentaro Moriichi (Asahikawa Medical University Hospital, Asahikawa); Masazumi Takahashi (Yokohama Municipal Citizen's Hospital, Yokohama); Yasutaka Takinishi, Takashi Sekikawa (Showa University Northern Yokohama Hospital, Yokohama); Naoki Okumura (Gifu University Hospital, Gifu); Shigemitsu Ueyama (Steel Memorial Hirohata Hospital, Himeji); Noriyuki Yamamura (Rinku General Medical Center, Izumisano); Dai Manaka (Kyoto-Katsura Hospital, Kyoto); Sachiko Oouchi (Hyogo Prefectural Kakogawa Medical Center, Kakogawa); Ryuichiro Ohashi (Kagawa Prefectural Central Hospital, Takamatsu); Tomohiko Mannami (Chugoku Central Hospital, Fukuyama); Kazuaki Tanabe (Hiroshima University Hospital, Hiroshima); Takeshi Shiraishi (Matsuyama Red Cross Hospital, Matsuyama).

\section{Compliance with ethical standards}

Funding This work was supported by the Japan Clinical Cancer Research Organization (JACCRO GC-06) with funding from Taiho Pharmaceutical Co., Ltd, Japan, under a research contract (no grant number).

Conflict of interest Yutaka Kimura: none declared. Masashi Fujii: none declared. Toshiki Masuishi received honoraria from Taiho Pharmaceutical and Chugai Pharma. Kazuhiro Nishikawa received honoraria from Taiho Pharmaceutical, Chugai Pharma, Yakult Honsha, and Ajinomoto. Chikara Kunisaki received donations from Yakult Honsha and Chugai Pharma. Yoshihiko Segawa received speakers bureau payments from Taiho Pharmaceutical, Eisai, Novartis, and Mochida Pharmaceutical as well as research funding from Taiho Pharmaceutical, Bayer Yakuhin, PAREXEL International, GlaxoSmithKline K.K., Daiichi Sankyo, and AstraZeneca. Japan. Masato Nakamura received honoraria from Merck Serono, Taiho Pharmaceutical, and Yakult Honsha. Kinro Sasaki: none declared. Narutoshi Nagao: none declared. Yukimasa Hatachi: none declared. Yasuhiro Yuasa: none declared. Shinya Asami: none declared. Masahiro Takeuchi received honoraria from Mitsubishi Tanabe Pharma and consulting fees from Hisamitsu Pharmaceutical, Kowa, Taiho Pharmaceutical, Shionogi Pharma, Abbvie, AstraZeneca Japan, and EA Pharma. Hiroshi Furukawa: none declared. Toshifusa Nakajima: none declared.

Open Access This article is distributed under the terms of the Creative Commons Attribution 4.0 International License (http://creativecommons.org/licenses/by/4.0/), which permits unrestricted use, distribution, and reproduction in any medium, provided you give appropriate credit to the original author(s) and the source, provide a link to the Creative Commons license, and indicate if changes were made. 


\section{References}

1. Ferlay J, Shin HR, Bray F, et al. Estimates of worldwide burden of cancer in 2008: GLOBOCAN 2008. Int J Cancer. 2010;127:2893-917.

2. Van Cutsem E, Moiseyenko VM, Tjulandin S, et al. Phase III study of docetaxel and cisplatin plus fluorouracil compared with cisplatin and fluorouracil as first-line therapy for advanced gastric cancer: a report of the V325 study group. J Clin Oncol. 2006;24:4991-7.

3. Cunningham D, Allum WH, Stenning SP, et al. Perioperative chemotherapy versus surgery alone for resectable gastroesophageal cancer. N Engl J Med. 2006;355:11-20.

4. Boku N, Yamamoto S, Fukuda H, et al. Fluorouracil versus combination of irinotecan plus cisplatin versus S-1 in metastatic gastric cancer: a randomised phase 3 study. Lancet Oncol. 2009;10:1063-9.

5. Koizumi W, Narahara H, Hara T, et al. S-1 plus cisplatin versus $\mathrm{S}-1$ alone for first-line treatment of advanced gastric cancer (SPIRITS trial): a phase III trial. Lancet Oncol. 2008;9:215-21.

6. Bang YJ, van Cutsem E, Feyereislova A, et al. Trastuzumab in combination with chemotherapy versus chemotherapy alone for treatment of HER2-positive advanced gastric or gastro-oesophageal junction cancer (ToGA): a phase 3, open-label, randomised controlled trial. Lancet. 2010;376:687-97.

7. Kurokawa Y, Sugimoto N, Miwa H, et al. Phase II study of trastuzumab in combination with S-1 plus cisplatin in HER-2 positive gastric cancer (HERBIS-1). Br J Cancer. 2014;110:1163-8.

8. Kris MG, Hesketh PJ, Somerfield MR, et al. American Society of Clinical Oncology guideline for antiemetics in oncology: update 2006. J Clin Oncol. 2006;24:2932-47.
9. Fillastre JP, Raguenez-Viotte G. Cisplatin nephrotoxicity. Toxicol Lett. 1989;46:163-75.

10. Arany I, Safirstein RL. Cisplatin nephrotoxicity. Semin Nephrol. 2003;23:460-4.

11. Koizumi W, Akiya T, Sato A, et al. Phase II study of S-1 as first-line treatment for elderly patients over 75 years of age with advanced gastric cancer: the Tokyo Cooperative Oncology Group study. Cancer Chemother Pharmacol. 2010;65:1093-9.

12. Gotoh M, Imamura $\mathrm{H}$, Takiuchi $\mathrm{H}$, et al. Phase II trial of S-1 for elderly patients (pts) over 75 years with advanced gastric cancer as first-line treatment (OGSG0404). Eur J Cancer Suppl. 2009;7(2):378.

13. Lim JH, Lee MH, Kim HG, et al. Three-weekly S-1 monotherapy as first-line treatment in elderly patients with recurrent or metastatic gastric cancer. Gut Liver. 2010;4:503-7.

14. NCI. Surveillance, Epidemiology, and End Results (SEER) database. http://seer.cancer.gov/csr/1795_2011). Accessed 13 Dec 2011.

15. Glimelius B, Ekstrom K, Hoffman K, et al. Randomized comparison between chemotherapy plus best supportive care with best supportive care in advanced gastric cancer. Ann Oncol. 1997;8:163-8.

16. Pyrhonen $\mathrm{S}$, Kuitunen $\mathrm{T}$, Nyandoto $\mathrm{P}$, et al. Randomised comparison of fluorouracil, epidoxorubicin and methotrexate (FEMTX) plus supportive care with supportive care alone in patients with non-resectable gastric cancer. Br J Cancer. 1995;71:587-91.

17. Murad AM, Santiago FF, Petroianu A, et al. Modified therapy with 5-fluorouracil, doxorubicin, and methotrexate in advanced gastric cancer. Cancer. 1993;72:37-41.

\section{Affiliations}

Yutaka Kimura ${ }^{1} \cdot$ Masashi Fujii $^{2} \cdot$ Toshiki Masuishi $^{3} \cdot$ Kazuhiro Nishikawa $^{4} \cdot$ Chikara Kunisaki $^{5} \cdot$ Satoshi Matsusaka $^{6}$. Yoshihiko Segawa ${ }^{7} \cdot$ Masato Nakamura $^{8} \cdot$ Kinro Sasaki $^{9} \cdot$ Narutoshi Nagao $^{10} \cdot$ Yukimasa Hatachi $^{11}$. Yasuhiro Yuasa ${ }^{12}$. Shinya Asami ${ }^{13}$. Masahiro Takeuchi ${ }^{14}$. Hiroshi Furukawa ${ }^{1} \cdot$ Toshifusa Nakajima $^{2}$ on behalf of the JACCRO GC-06 Study Group

1 Department of Surgery, Kindai University Faculty of Medicine, Osaka-Sayama, Japan

2 Japan Clinical Cancer Research Organization, 7F Ginza Wing Building, 1-14-5, Ginza, Chuo-ku, Tokyo 104-0061, Japan

3 Department of Clinical Oncology, Aichi Cancer Center Hospital, Nagoya, Japan

4 Department of Surgery, Osaka National Hospital, Osaka, Japan

5 Department of Surgery, Gastroenterological Center, Yokohama City University, Yokohama, Japan

6 Department of Gastroenterology, Cancer Institute Hospital of the Japanese Foundation for Cancer Research, Tokyo, Japan

7 Department of Medical Oncology, Saitama Medical University International Medical Center, Hidaka, Japan
8 Aizawa Comprehensive Cancer Center, Aizawa Hospital, Matsumoto, Japan

9 First Department of Surgery, Dokkyo Medical University Hospital, Tochigi, Japan

10 Department of Surgery, Gifu Prefectural General Medical Center, Gifu, Japan

11 Department of Medical Oncology, Kobe City Medical Center General Hospital, Kobe, Japan

12 Department of Surgery, Tokushima Red Cross Hospital, Tokushima, Japan

13 Department of Surgery, Fukuyama City Hospital, Fukuyama, Japan

14 Department of Clinical Medicine (Biostatistics), Kitasato University School of Pharmacy, Tokyo, Japan 